Landslides (2018) 15:1437-1441 DOI 10.1007/s10346-018-0976-2 Received: 10 October 2017 Accepted: 13 March 2018 Published online: 28 March 2018 (c) The Author(s) 2018
Francesca Bozzano - Paolo Mazzanti - Serena Moretto

\section{Discussion to: 'Guidelines on the use of inverse velocity method as a tool for setting alarm thresholds and forecasting landslides and structure collapses' by T. Carlà, E. Intrieri, F. Di Traglia, T. Nolesini, G. Gigli and N. Casagli}

\begin{abstract}
The paper 'Guidelines on the use of inverse velocity method as a tool for setting alarm thresholds and forecasting landslides and structure collapses' by T. Carlà, E. Intrieri, F. Di Traglia, T. Nolesini, G. Gigli and N. Casagli deals with a sensitive topic for landslide risk management. Exploring the pre-failure behaviour of four different case histories, the authors proposed standard procedures for the application of the inverse velocity method (INV, Fukuzono 1985). Specifically, they suggested guidelines for the filtering of velocity data and an original and simple approach to automatically set the first and the second alarm thresholds using the inverse velocity method. The present discussion addresses three different topics: (1) data filter selection according to the features of monitoring instrument; (2) the importance of data sampling frequency for the forecasting analysis and (3) the influence of the starting point (SP in this discussion) for the application of INV analysis. Moreover, based on this matter, a new method is proposed to update the INV analysis on an ongoing basis.
\end{abstract}

Keywords Landslide forecasting · Inverse velocity method $\cdot$ Starting point

The application of INV analysis was also investigated in a paper recently published in this journal (Mazzanti et al. 2014). This discussion is a good occasion to compare the results obtained in the two papers where a similar approach was applied to different case histories.

In Mazzanti et al. (2014), small and shallow landslides were monitored with high spatial and temporal resolution; on the contrary, in Carlà et al. (2016), monitoring data characterised by a lower sampling frequency of larger and deeper slopes and wall collapse are presented (Mt. Beni and Vajont landslides, Stromboli talus and Volterra wall). Specifically, in Mazzanti et al. (2014), prefailure time series of displacement inferred from TInSAR monitoring (Terrestrial Synthetic Aperture Radar Interferometry) of ten small-scale rockslides were explored.

For the ten shallow landslides, the INV analysis was performed using a moving-average approach similar to the one adopted by Carlà et al. (2016). Even though the cases studied in the two papers and the features of the monitoring instruments are very different, the same results were obtained: data filtering allowed better performance of the INV analysis.

Topic 1. Data filter selection

Carlà et al. introduced general rules to identify a suitable data filtering procedure, according to the deformational behaviour of the slope. They suggest avoiding strong data filtering when the condition of $\alpha=2$ is satisfied and for a high rate of movement (because filters could affect the sensitivity for detecting deformation-trend changes without leading to important improvements to the INV analysis). On the other hand, if the landslide is characterised by low movement rates, Carlà et al. stated that data smoothing can considerably improve the results of the INV analysis (because monitoring data can be significantly affected by instrumental noise).

We agree with Carlà et al. (2016) that the slope behaviour must influence the type of data filtering. Here, we also want to note the role played by the features of the monitoring instrument for the selection of a suitable data filter. Specifically, considering the TInSAR monitoring technique, instrumental noise (particularly atmospheric noise) could strongly affect the results of INV analysis (Mazzanti et al. 2014, Dick et al. 2014), irrespective of the deformational behaviour of a slope.

Figure 1 shows the comparison between the INV method applied on a time series of displacement inferred from the TInSAR technique (namely, IBIS-L terrestrial SAR interferometer, by IDS S.p.A) and on the filtered time series. Pre-failure monitoring data refer to the small-scale landslide that occurred on the 24 January 2009 (i.e. landslide number 1 in Mazzanti et al. 2014).

Thanks to the high temporal resolution of data collection (approximately $5 \mathrm{~min}$ ), a direct-form FIR equiripple low-pass filter has been implemented in the Matlab environment. The filter significantly improved the signal-to-noise ratio and the quality of the time series (Fig. 1a). In Fig. 1, the INV analysis has been applied simulating the data acquisition over time. The results show that the digital filter improves both the accuracy and the reliability of the INV method. Indeed, more accurate and more stable predictions have been obtained using the filtered dataset. Furthermore, using filtered data, the linear regression is characterised by very high $\mathrm{R}$-squared $\left(R^{2}\right)$ values during all the analysis (Fig. 1c).

Mazzanti et al. (2014) showed that to obtain reliable results with the INV analysis, post processing of time series of displacement was necessary for all ten landslides analysed. After data filtering through the FIR equiripple low-pass filter, the movingaverage approach further improved the predictions (Mazzanti et al. 2014, Fig. 13). According to the data presented in Fig. 1 and Mazzanti et al.'s 2014 results, and considering the TInSAR characteristics (i.e. the high data sampling frequency and the instrumental noise), we conclude that a double data filtering can be used to improve the stability of INV analysis, without losing the sensitivity to follow different displacement trends. 


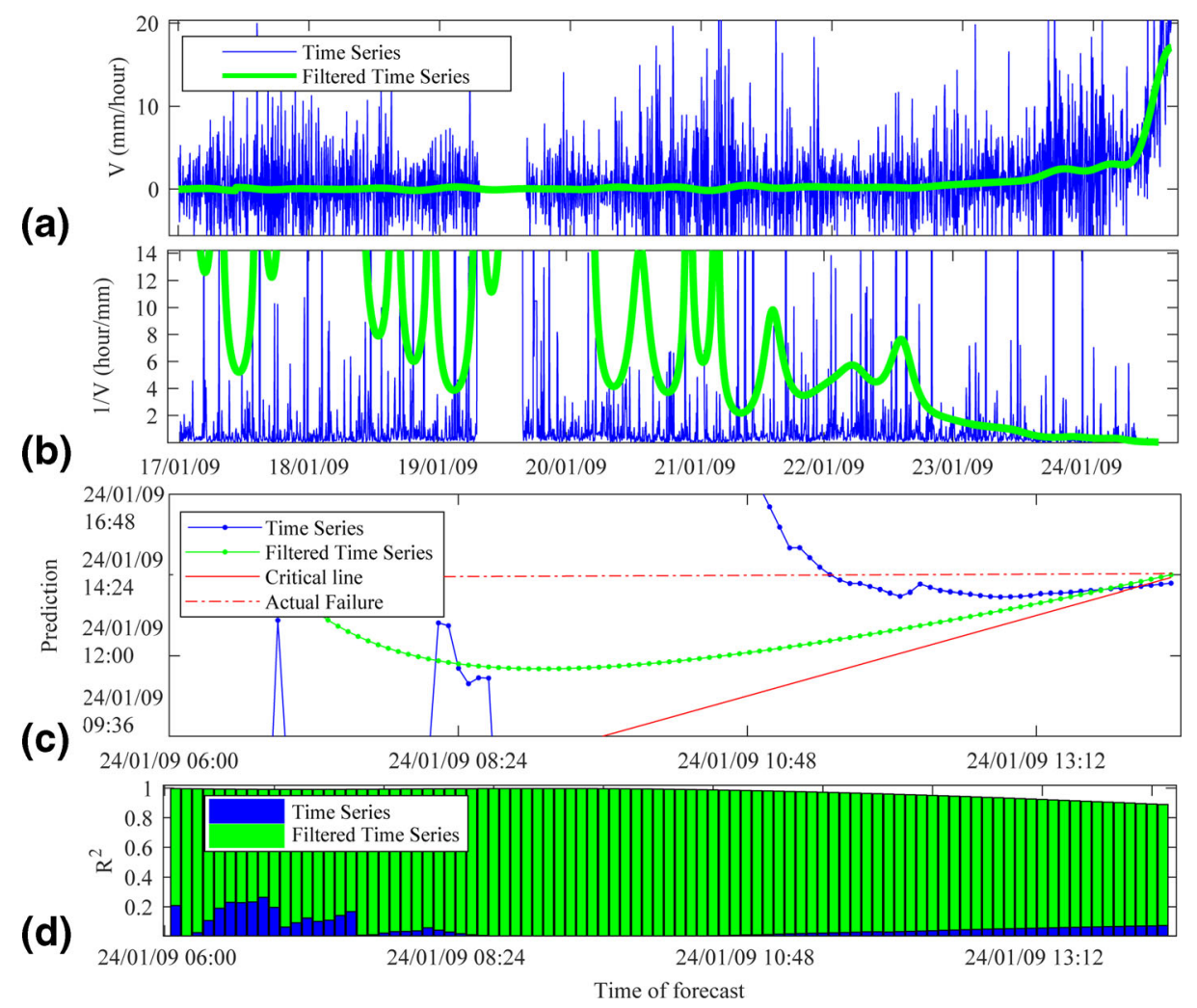

Fig. 1 Comparison between INV analysis applied on not-filtered time series of velocity and on the corresponding filtered time series. a Velocity datasets. b Inverse velocity plot. $\mathrm{c}$ Results of the INV analysis. The $\mathrm{x}$-axis represents the time when the forecast is made, while the predicted time of failure is reported on the $y$-axis. The critical line represents the lower bound of a reasonable prediction, as it corresponds to the day when the forecast is made. $\mathbf{d}$ R-squared $\left(R^{2}\right)$ values of the best-fit linear model in a least-squares sense for both filtered and not-filtered time series

Topic 2. The role of data sampling frequency

In Mazzanti et al. (2014), the high frequency of data acquisition of TInSAR allowed prediction of the ToF $\left(\mathrm{T}_{\mathrm{f}}\right.$ in Carlà et al. (2016)) of the ten small-scale landslides, characterised by a short accelerating period ranging between 370 and $12 \mathrm{~h}$.

The sampling frequency is relevant especially for slopes characterised by a short acceleration phase prior to the collapse. Furthermore, with higher sampling frequency, more predictions can be performed once the OOA (onset of acceleration, Dick et al. 2014) or a change of trend is detected. For example, considering the Volterra wall case study presented by Carlà et al (2016), a monitoring device with a higher sampling frequency would probably have allowed them to perform the INV analysis. The time series reported in the velocity-time space (Fig. 13a in Carlà et al. 2016) shows a change in the trend curve corresponding to the 1 March. Only a few data were acquired between the 1 March and the wall collapse, not allowing recognition of a well-defined linear trend in the $1 / \mathrm{V}$-time space during the terminal phase. If data were collected every $5 \mathrm{~min}$, a better prediction would have been possible. To support this statement, we applied the INV analysis to the Volterra case study simulating a higher sampling frequency (Fig. 2). The Volterra-wall monitoring data presented by Carlà et al. in Fig. 13a have been digitised using the Matlab code 'grabit'. Then, these data have been oversampled according to a sampling interval of $5 \mathrm{~min}$ (Fig. 2a), and the INV analysis has been applied on the modified dataset. The starting point (SP) of the analysis is highlighted with the black arrow in Fig. 2a. Figure 2a shows that the accuracy of the predictions increases approaching the actual failure following a linear trend. At the same time, a decrease of the $R^{2}$ value can be observed. It is worth highlighting that the decrease in the $R^{2}$ value is associated with the deviation of data from the linear model. Indeed, a decrease in the velocity trend can be observed in the last data points (from the 1 March to the collapse), producing regression lines with lower $R^{2}$. We can conclude that data sampling frequency is a key factor for landslide risk management and for the application of INV analyses in real situations. In fact, the analysis presented here suggests that it would have been possible to apply the forecasting analysis for the Volterra case study making use of datasets with higher sampling frequency.

Topic 3. The influence of the starting point (SP) on the INV analysis Another important factor influencing the application of forecasting methods is the starting point (SP) of the analysis or rather, the point when the INV analysis is started.

Carlà et al. (2016) provided a simple and innovative method to identify the onset of acceleration (i.e. the beginning of the acceleration phase and the point at which the INV analysis should start) or the trend update points (i.e. the points marking a change in the deformation trend as stated by Dick et al. 2014). They defined the OOA/TU as a 'positive crossover'; namely, the point where the short-term moving-average (SMA) line crosses above the longterm moving-average (LMA) line. The method is interesting and 

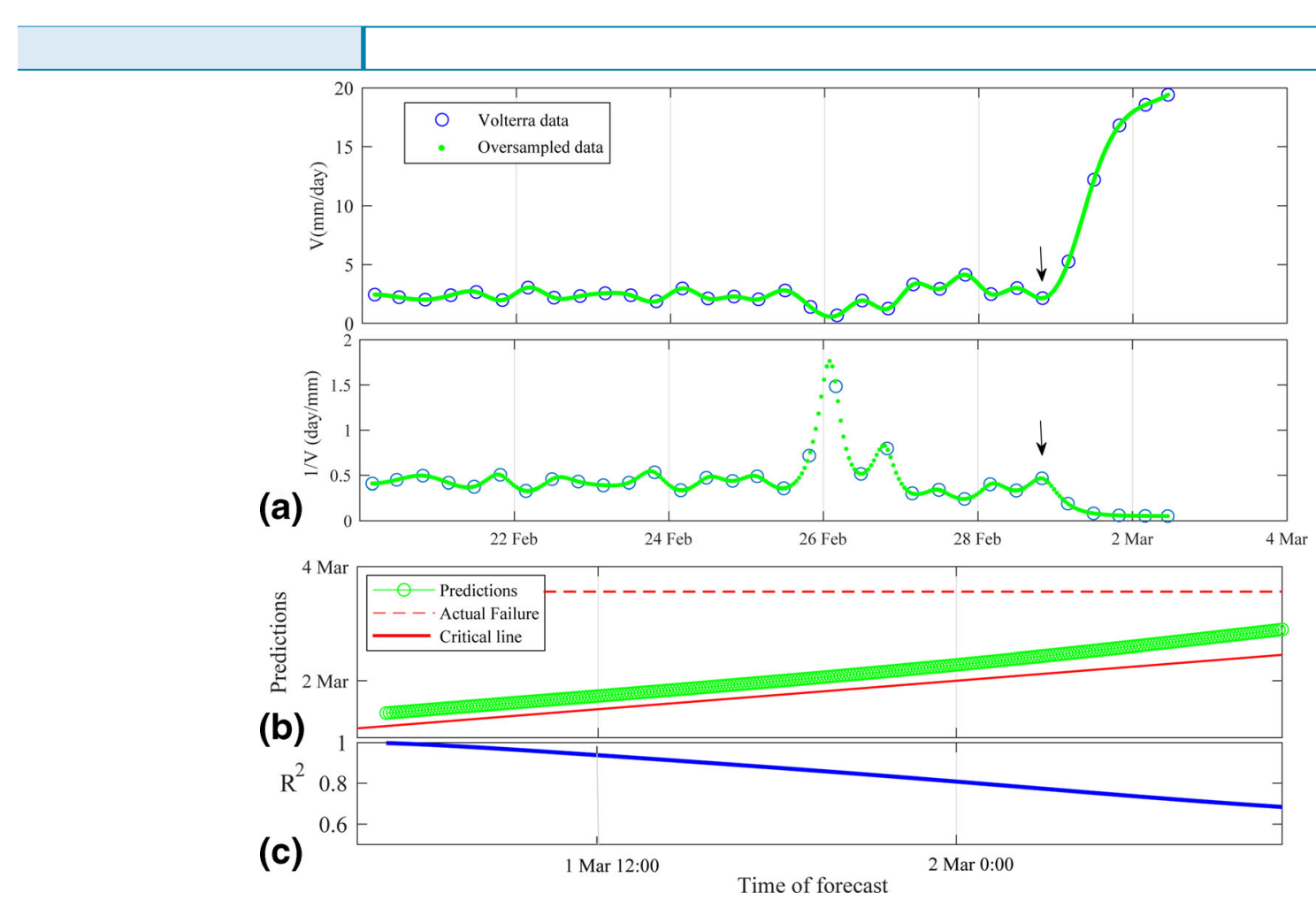

Fig. 2 INV analysis applied on oversampled Volterra's monitoring data. a Volterra data and oversampled time series in the velocity vs time space. b Oversampled time series in the inverse velocity vs time space. $\mathbf{c}$ Results of the INV analysis. $\mathbf{d} R^{2}$ associated with each prediction

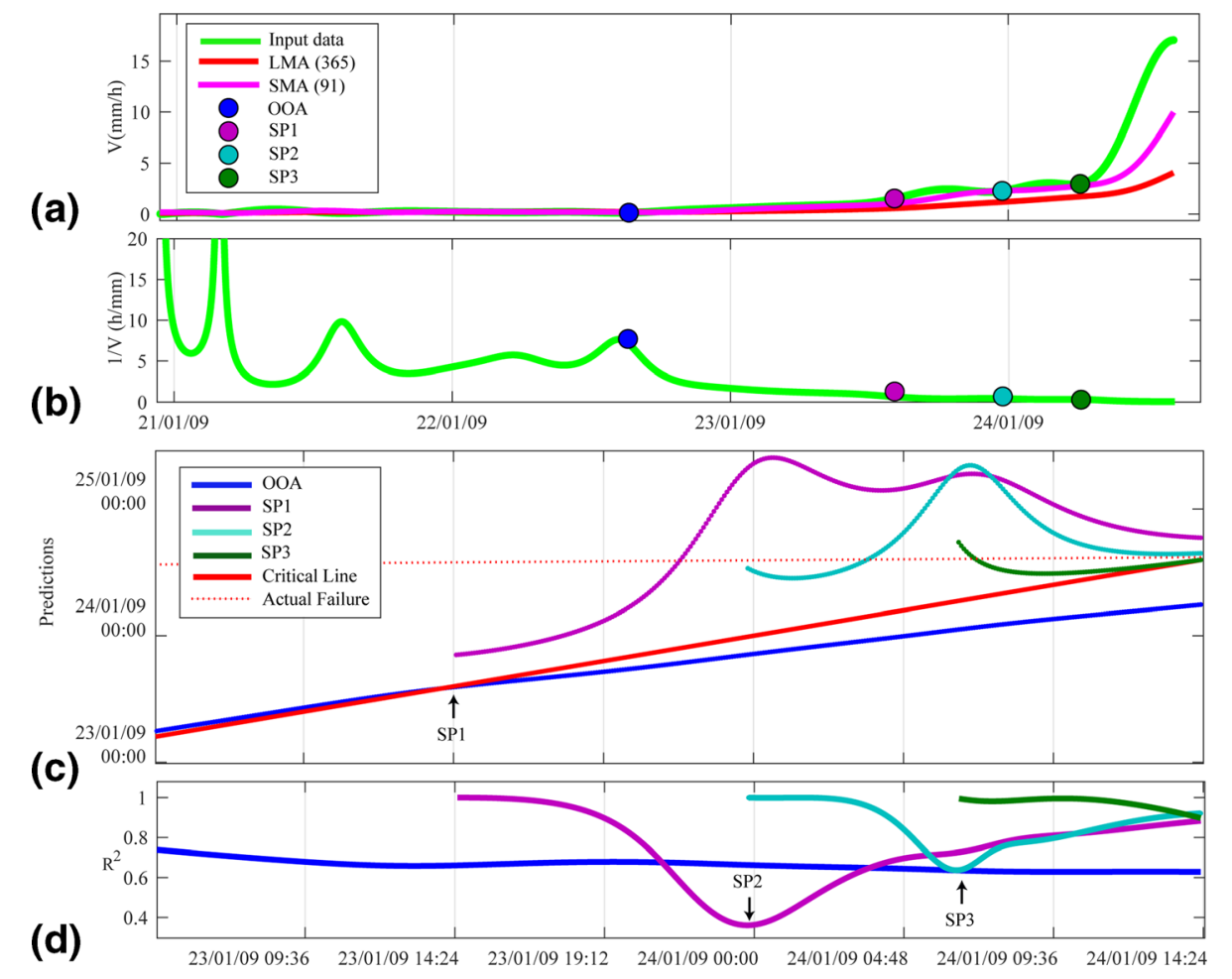

Fig. 3 INV analysis by the updating of the SPs. The dataset used corresponds to the filtered one shown in Fig. 1 (i.e. landslide number 1 in Mazzanti et al. 2014). a Velocity data (green line), short-term and long-term moving average (SMA, LMA). The SPs of the INV analysis are reported with different colours. The 00A has been defined as the positive crossover between SMA and LMA lines. $\mathbf{b}$ Inverse velocity data. c Predictions according to each SP. $\mathbf{d} R^{2}$ related to each SP. 


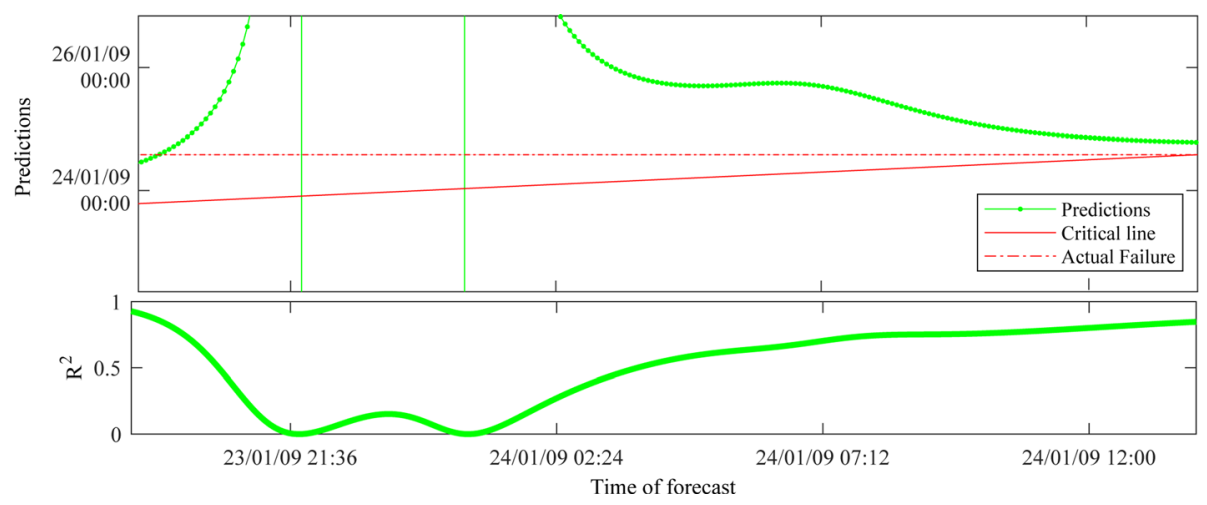

Fig. 4 Relation between the variation of the prediction time and the $R^{2}$

useful because at present, there is not a standard procedure for the application of the Fukuzono analysis and for the identification of the beginning of the acceleration phase. The INV method is based on the identification of a consistent linear trend; once a first OOA has been identified, possible trend changes due to different controlling factors can occur, and to obtain consistent predictions, they must be taken into account and the predictions promptly revised (Rose and Hungr 2007).

Furthermore, the SP of the INV analysis is extremely important because it strongly affects the results. In fact, by changing the SP, very different predictions are obtained (Fig. 3).

For these reasons, we propose a new method to update the INV analysis in order to increase the prediction accuracy with the collection of new monitoring data. This method is based on updating the INV analysis on an ongoing basis through the identification of suitable new SPs. Once a first OOA/TU has been identified, the new SPs can be selected according to the following criteria: (i) reasonability of predictions; (ii) $R^{2}$ value; and (iii) stability of predictions. A prediction is defined unreasonable if it is below the critical line. On the other hand, it is defined unstable if the computed ToF is characterised by large, rapid and random variations (e.g. predictions in Fig. $1 \mathrm{~b}$ using the unfiltered time series). Indeed, in our view, unstable predictions testify the unreliability of the analysis. Furthermore, they cannot be managed in real emergency scenarios. Additionally, $R^{2}$ is considered an indicator of the reliability of the INV analysis. In fact, it quantifies the agreement between the monitoring data and the INV model, so that a trend change is represented by a decrease in the $R^{2}$ value. In addition, a direct relationship between the $R^{2}$ value and the stability of predictions can be observed in Fig. 4, where low $R^{2}$ values correspond to large changes in the predictions. The relation between the correlation coefficient and the reliability of INV analysis is also shown by Manconi and Giordan (2014) for the case of study of La Saxe landslide.

Hereafter, using the pre-failure monitoring data reported in Fig. 1 (i.e. landslide number 1 in Mazzanti et al. 2014), an example of the application of our method to update the predictions is shown and explained (Fig. 3). The first step is the identification of the OOA. To this aim, the 'crossover method', proposed by Carlà et al. (2016), has been used. A window of 365 data for the LMA and 91 for SMA (Fig. 3a) has been employed. SMA's window has been chosen according to 1/15 of the dataset's length, while LMA is four times the SMA. Starting from the OOA, the INV iterative analysis has been performed (blue line Fig. 3b). On 23/01/2009 at 14:0o Central European Time (CET), the prediction intersects the critical line (Fig. 3b); from that time until the occurrence of failure, no reliable predictions have been obtained (i.e. all the predictions are below the critical line). In such a case, a new SP is necessary to perform INV analysis and obtain reliable results. Thus, the point after the intersection has been selected as the new SP (SP1). Starting a new INV analysis from SP1, reasonable predictions have been obtained (violet line Fig. $3 \mathrm{~b}$ ), and the best-fit has been improved (violet line Fig. 3c). Nevertheless, the $R^{2}$ value starts to decrease abruptly on 23/01/2009 at 19:12 CET, related to a change in the velocity trend. As soon as the $R^{2}$ value starts to increase, the new $\mathrm{SP}_{2}$ has been selected. Using $\mathrm{SP}_{2}$, a sudden increase in the accuracy of predictions can be observed, as well as an improvement of the fit between the Fukuzono linear regression and the monitoring data (cyan line Fig. 3b, c). For the selection of the $\mathrm{SP}_{3}$, the same criterion has been used, thus allowing attainment of a very high accuracy in the time of failure estimation (green line predictions Fig. 3b) and a very high correlation between the monitoring data and regression line (green line Fig. 3c).

The here-proposed method is very sensitive to the trend changes, allowing to identify also the slight variations of deformation trends. Furthermore, the methodology also considers the features of monitoring data, which can influence the accuracy and the reliability of the INV analysis. In fact, while the crossover method allows to identify the onset of an uptrend, the need to change the SP can be also related to the monitoring data features. For example, natural and/or instrumental noise in the time series can lead to the decrease of both R-squared values and stability of predictions, thus requiring the selection of a new SP in order to improve the reliability of the INV analysis. Based on our analysis, we conclude that once a first OOA has been identified, it is strongly recommended to update the INV analysis if at least one of the following conditions is verified: (i) the predictions are below the critical line; (ii) the $R^{2}$ shows a rapid decrease; or (iii) unstable predictions are obtained.

Open Access This article is distributed under the terms of the Creative Commons Attribution 4.0 International License (http:// creativecommons.org/licenses/by/4.o/), which permits unrestricted use, distribution, and reproduction in any medium, provided you give appropriate credit to the original author(s) and the source, provide a link to the Creative Commons license, and indicate if changes were made. 


\section{References}

Carlà T, Intrieri E, Di Traglia F, Nolesini T, Gigli G, Casagli N (2016) Guidelines on the use of inverse velocity method as a tool for setting alarm thresholds and forecasting landslides and structure collapses. Landslide 14(2):517-534

Dick GJ, Eberhardt E, Cabrejo-Liévano AG, Stead D, Rose ND (2014) Development of an early-warning time-of-failure analysis methodology for open-pit mine slopes utilizing ground-based slope stability radar monitoring data. Can Geotech 52:515-529

Fukuzono T (1985) A new method for predicting the failure time of slopes. Proceedings, 4th International Conference \& Field Workshop on Landslides, Tokyo, pp 145-150

Manconi A, Giordan D (2014) Landslide failure forecast in near-real-time. Geomat Nat Haz Risk 7:639-648. https://doi.org/10.1080/19475705.2014.942388

Mazzanti P, Bozzano F, Cipriani I, Prestininzi A (2014) New insights into the temporal prediction of landslides by a terrestrial SAR interferometry monitoring case study. Landslides. http://link.springer.com/article/10.1007\%2Fs10346-014-0469-x
Rose ND, Hungr 0 (2007) Forecasting potential rock slope failure in open pit mines using the inverse-velocity method. Int J Rock Mech Min Sci 44:308-320

F. Bozzano (四) · P. Mazzanti · S. Moretto

Department of Earth Sciences,

Sapienza Università di Roma,

P.le Aldo Moro 5, 00185, Roma, Italy

Email: francesca.bozzano@uniroma1.it

\section{P. Mazzanti}

NHAZCA S.r.l., spin-off of "Sapienza" University of Rome,

Via V. Bachelet 12, Rome, Italy 